\title{
A Dual-modular Aptasensor for Detection of Cardiac Troponin I Based on Mesoporous Silica Films by Electrochemiluminescence / Electrochemical Impedance Spectroscopy
}

\author{
Xiaona Mi, ${ }^{\perp, \S}$ Hui Li, ${ }^{\perp, \dagger}$ Rong Tan ${ }^{\S}$ and Yifeng $\mathrm{Tu}^{\S, 1 *}$ \\ $\S$ College of Chemistry, Chemical Engineering and Material Science, Soochow \\ University, Suzhou, 215123, P. R. China \\ 'Department of Cardiology, The Second Affiliated Hospital of Soochow University, \\ Suzhou, 215004, P. R. China \\ *Corresponding author: Prof. Tu Yifeng, PhD. \\ E-mail address: tuyf@suda.edu.cn
}

Table of contents

S1. The challenge of COVID-19 pandemic---------------------------------------------S-2

S2. Destructiveness of false results-------------------------------------------------------S-3

S3. Instruments and reagents--_4

S4. The preparation of MSF on ITO 5

S5. The comparison of sensing performance with reported ones----------------S-6

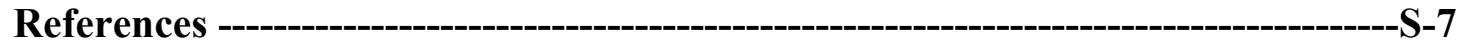




\section{S1. The challenge of COVID-19 pandemic}

Began in the end of last year, a worldwide pandemic of COVID-19 has arisen. It is seriously threatening the health and safety of citizens, meanwhile it resulted in tremendous economic and social problems. Until now, a global mortality of $3.26 \%$ (in 27.5 millions of confirmed cases, WHO, https://covid19.who.int/) intimidates those confirmed COVID-19 persons. Myocardial injury has been noted in a significant number of infected COVID-19 patients. ${ }^{1,2}$ Moreover, those biomarkers for cardiac injury especially the cTnI, which notably increased in the blood of those COVID-19 severe patients. In one of the initial studies in Wuhan, Huang et al reported increased troponin I levels (> $28 \mathrm{pg} / \mathrm{ml}$ ) in 5 of 41 COVID-19 patients, in whom the diagnosis of virus-related cardiac injury was made. In their study, 4 out of 5 patients with elevated cTnI required ICU admission. ${ }^{3}$ Recent studies have reported myocardial injury in patients with COVID-19 and suggested an association between myocardial injury and mortality. ${ }^{2,4,5}$ Guo et al reported a higher in-hospital crude mortality in patients with myocardial injury than in patients without myocardial injury $(59.6 \%$ vs. $8.9 \%)$ in a study of 187 patients with COVID- $19^{4}$ and found a stepwise increase in mortality when they split the study population into subgroups of patients according to the presence of pre-existing cardiovascular disease alone, myocardial injury alone, or their combination $\left(13.3 \%, 37.5 \%\right.$, and $69.4 \%$, respectively). ${ }^{4}$ Shi et al also found that myocardial injury was independently associated with mortality, with an adjusted HR of $4.26(\mathrm{P}<0.001)$, in a study of 416 patients with COVID-19. ${ }^{5}$ Acute respiratory distress syndrome (ARDS) is another independent risk factor for mortality with COVID-19. ARDS occurs as a result of an acute systemic inflammatory response, which can be caused by insults to the lung, either direct or indirect. ${ }^{6}$ Liu et al reported on an 85-year-old Chinese male who died following COVID-19 infection? ${ }^{7}$. The autopsy report pointed out that the copious amounts of gray-white viscous fluid was found to in the alveoli without air exchange, causing oxygen deficiency. Myocardial infarction caused by oxygen deficiency, which in turn causes the rise of biomarkers of myocardial infarction. So, to provide more effective method to monitor the cardiac function is significant for therapy of COVID-19 patients. 


\section{S2. The destructiveness of false results}

The possible false result is the common defect of some analytical methods especially in clinical field. The false result will lead to mistakes in clinical diagnosis, which will result in two severe problems: 1) The patients will lose their chance of therapy even pay the price of life; 2) Excessive medical care not only wastes medical and economic resources, but also may cause physical and mental harm to patients. False negatives mainly originated from insufficient sensitivity of methods whereas false positives might come from the deficiency in their anti-interference performance. Therefore, our aim is: 1) To comprehensively improve the sensitivity and specificity of the biosensor, which is the essential base of whole effort; 2) To provide timely measures to find possible false results, so that the greatest degree of quality assurance can be achieved in the testing process. In summarization, more accurate detection and lower false rate is an important challenge for medical diagnosis.

In this work, the detection of cTnI concentration in blood samples is a key factor in the evaluation of patients with the risk of acute myocardial infarction (AMI). The early diagnosis and intervention could save the lives, or else the patients might face the death in days even hours. But the false results will lead to improper judgement and treatment. Hence, to avoid false results for cTnI detection is very necessary to get optimal benefit. To develop a biosensor with higher sensitivity, good anti-interference ability and exact accuracy is the ultimate goal of our research to fulfil abovementioned idea. To achieve it, we designed and fabricated a dual-modular biosensor which possesses all of those essential factors. Beside the ideal performances of sensitivity and specificity, we achieved the dual-modular output from single biosensor with different probe, which can provide the opportunity to avoid false results by self-verification for accurate detection of cTnI. It should be pointed out, the detection techniques employed in this sensor have some particularities. The responses of ECL or EIS toward target are just opposite, which give the best collocation for self-verification because of the complementarity between them. The self-verification will be adopted as a manner of quality control in real application by the program of random inspection, thus to implement a bypassing supervision to ensure the accuracy of results. 


\section{S3. Instruments and reagents}

ECL experiments were conducted on a lab-built apparatus. The transmission electron microscopy (TEM) images were obtained on a Tecnai G20 (FEI, USA) instrument, for observing the morphology of mesoporous silica film. The Dimension Icon atomic force microscopy (AFM) (Bruck, Germany) was used to measure the thickness of MSF. A UV-3600 UV-Vis spectrophotometer (Shimadzu, Japan) was used to explore the formation of the imine bond between glutaraldehyde and amino-aptamer. FT-IR spectra were recorded by Bruker V70+ HYPERON1000 spectrometer (Ettlingen, Germany). XPS photoelectron spectra were obtained on an EXCALAB 250 XI spectrometer with Ag K $\alpha$ X-ray (Thermo Scientific, U.S.). Energy dispersive X-ray spectroscopy (EDX) measurement was done on a SU8010 microscope with an accelerating voltage of $20 \mathrm{kV}$ (Hitachi, Japan). The XRD data for MSF were collected by X'Pert-Pro MPD (Panalytical, Holland), using $\mathrm{Cu} \mathrm{K \alpha}(\lambda=1.5406 \AA)$ as radiation source. The static contact angle tests were carried out on a DATAPHYSICS contact angle system (OCAT2, Germany). The cyclic voltammetry (CV) and electrochemical impedance spectroscopy (EIS) were achieved with an RST-5200 electrochemical workstation (Risetest Electronic Co., Ltd. Suzhou, China). The ITO is supplied by Guluo Glass Co., Ltd. (Henan, China). The electrochemical cell was conducted with the traditional threeelectrode system, consisted of a bare or functioned ITO (the effective area of $1 \mathrm{~cm} \times 1 \mathrm{~cm}$ ) working electrode, a platinum wire counter electrode and a saturated calomel reference electrode (SCE).

The $\mathrm{NH}_{2}$-cTnI-Tro4 aptamer is synthesized in Sangon Biotech Co., Ltd. (Shanghai, China) sequenced as: ${ }^{8} 5^{\prime}-\mathrm{NH}_{2}-\left(\mathrm{CH}_{2}\right)_{6}$-CGT GCA GTA CGC CAA CCT TTC TCA TGC GCT GCC CCT CTT A-3'. Recombinant human cardiac troponin I protein is purchased from Abcam (Cambridge, UK). Tetraethoxysilane (TEOS, $98.5 \%$ ), glutaraldehyde (GA, $25 \%$ ), cetyltrimethyl-ammonium bromide (CTAB) and 3-aminopropyltrimethoxysilane (APTMS) are bought from Sinopharm Chemical Reagent Co., Ltd. (Shanghai, China). Luminol is provided by Fluka (USA). All other chemicals are analytical grade without further purification. All aqueous solutions are prepared using ultrapurified water (18.2 $\mathrm{M} \Omega \mathrm{cm}$ ). $0.1 \mathrm{M}$ phosphate buffer solution (pH 7.4) containing $10 \mathrm{mM} \mathrm{NaCl}, 5$ $\mathrm{mM} \mathrm{KCl}$ and $1 \mathrm{mM} \mathrm{MgCl} 2$ is used for the preparation of $\mathrm{NH}_{2}$-Tro4 Apt and cTnI solutions. Human serum samples are provided by the Second Affiliated Hospital of Soochow University (Suzhou, China), stored under $-20^{\circ} \mathrm{C}$. 


\section{S4. The preparation of MSF on ITO}

The ITO glass was firstly soaked in $0.5 \mathrm{M} \mathrm{NaOH}$ solution of ethanol at room temperature overnight in order to remove most of surface organic residues, then cleaned with acetone and water in sequence for 30 min under ultrasonication, and then dried under a nitrogen stream. The vertically well-defined MSF was grown in situ on ITO by the Stöber-solution method as previously reported.

Briefly, it was brought about by submerging ITO in a mixture solution made of CTAB (0.16 g), ethanol (30 mL), water $(70 \mathrm{~mL}), 10 \mu \mathrm{L}$ of ammonia water (25 wt \%) and $80 \mu \mathrm{L}$ of TEOS, in quiescence for $24 \mathrm{~h}$ at $60^{\circ} \mathrm{C}$. The CTAB-templated silica film is thus deposited on the ITO substrate, it was then flushed with water, dried with $\mathrm{N}_{2}$ and further aged at $100{ }^{\circ} \mathrm{C}$ in an oven overnight. Finally, treated with $0.1 \mathrm{M} \mathrm{HCl} /$ ethanol solution for $30 \mathrm{~min}$ under mild stirring to extract those CTAB-micelles to empty those nanochannels, a hydrophilic MSF is formed on ITO. After that, it was soaked in $2 \mathrm{~mL}$ of $5 \% 3$-aminopropyltrimethoxysilane (APTMS) ethanol solution for $12 \mathrm{~h}$ at room temperature to guarantee the amino-functionalization of the mouth margin of those nanochannels. ${ }^{9}$ 


\section{S5. The comparison of sensing performance with reported ones}

Table S1. Comparison of the performances of this aptasensor towards cTnI with reported biosensors.

\begin{tabular}{|c|c|c|c|}
\hline Methods & Nanomaterials & $\begin{array}{c}\text { LOD } \\
\left(\text { pg mL } L^{-1}\right)\end{array}$ & $\begin{array}{l}\text { Linear range } \\
\left(\text { ng } \mathrm{mL}^{-1}\right)\end{array}$ \\
\hline Fluorescene & $\mathrm{FMGC}^{a}$ & 0.1 & $0.1-100$ \\
\hline $\mathrm{ECL}^{b}$ & FTO/nGO@ $\mathrm{TiO}_{2} \mathrm{NLPs}^{c}$ & 0.96 & $0.0024-2.4$ \\
\hline Colorimetric & peptide-functionalized AuNPs & 200 & $0.1-500$ \\
\hline Electrochemical & $\mathrm{GCE} / \mathrm{PrGO}^{d}$ & 70 & $0.1-10$ \\
\hline $\mathrm{PEC}^{e}$ & $\begin{array}{c}\mathrm{ITO} / \mathrm{Zn}_{2} \mathrm{SnO}_{4} / \mathrm{N}, \mathrm{S}- \\
\mathrm{GQDs} \mathrm{CdS}^{f} / \mathrm{CdS}\end{array}$ & 0.3 & $0.001-50$ \\
\hline ECL/EIS & ITO/amino-MSF & $\begin{array}{l}\text { EIS } 0.05 \\
\text { ECL } 0.02\end{array}$ & $0.00005-1$ \\
\hline
\end{tabular}

${ }^{a}$ FMGC: fluoro-microbead guiding chip. ${ }^{b} \mathrm{ECL}$ : Electrochemiluminescence.

${ }^{c} \mathrm{FTO} / \mathrm{nGO} @ \mathrm{TiO}_{2}$ NLPs: F-doped tin oxide electrode coated with nanographene oxide wrapping titanium dioxide.

${ }^{d} \mathrm{GCE} / \mathrm{PrGO}$ : glassy carbon electrode/ porous graphene oxide.

${ }^{e}$ PEC: photoelectrochemical. ${ }^{\mathrm{N}}, \mathrm{S}-\mathrm{GQD}$ : graphene quantum dots doped with nitrogen and sulfur. 


\section{REFERENCES}

(1) Babapoor-Farrokhran, S.; Gill, D.; Walker, J.; Rasekhi, R. T.; Bozorgnia, B.; Amanullah, A. Life Sciences 2020, 253.

(2) Ferrante, G.; Fazzari, F.; Cozzi, O.; Maurina, M.; Bragato, R.; D'Orazio, F.; Torrisi, C.; Lanza, E.; Indolfi, E.; Donghi, V.; Mantovani, R.; Liccardo, G.; Voza, A.; Azzolini, E.; Balzarini, L.; Reimers, B.; Stefanini, G. G.; Condorelli, G.; Monti, L. Cardiovascular research $\mathbf{2 0 2 0 .}$

(3) Huang, C. L.; Wang, Y. M.; Li, X. W.; Ren, L. L.; Zhao, J. P.; Hu, Y.; Zhang, L.; Fan, G. H.; Xu, J. Y.; Gu, X. Y.; Cheng, Z. S.; Yu, T.; Xia, J. A.; Wei, Y.; Wu, W. J.; Xie, X. L.; Yin, W.; Li, H.; Liu, M.; Xiao, Y., et al. Lancet 2020, 395, 497-506.

(4) Guo, T.; Fan, Y.; Chen, M. Jama Cardiology 2020, 5, 848-848.

(5) Shi, S. B.; Qin, M.; Shen, B.; Cai, Y. L.; Liu, T.; Yang, F.; Gong, W.; Liu, X.; Liang, J. J.; Zhao, Q. Y.; Huang, H.; Yang, B.; Huang, C. X. Jama Cardiology 2020, 5, 802810.

(6) Li, X.; Ma, X. Crit Care 2020, 24, 198.

(7) Liu, Q.; Wang, R. S.; Qu, G. Q.; Wang, Y. Y.; Liu, P.; Zhu, Y. Z. e. a. Jounal of Forensic Medicine 2020, 36, 21-23.

(8) Jo, H.; Gu, H.; Jeon, W.; Youn, H.; Her, J.; Kim, S. K.; Lee, J.; Shin, J. H.; Ban, C. Anal. Chem. 2015, 87, 9869-9875.

(9) Zhao, M. J.; Wu, W. H.; Su, B. ACS Appli. Mater. Interfaces 2018, 10, 33986-33992.

(10) Song, S. Y.; Han, Y. D.; Kim, K.; Yang, S. S.; Yoon, H. C. Biosens. Bioelectron. 2011, 26, 3818-3824.

(11) Han, Z.; Shu, J.; Liang, X.; Cui, H. Anal. Chem. 2019, 91, 12260-12267.

(12) Liu, X. H.; Wang, Y.; Chen, P.; McCadden, A.; Palaniappan, A.; Zhang, J. L.; Liedberg, B. ACS Sens. 2016, 1, 1416-1422.

(13) Kazemi, S. H.; Ghodsi, E.; Abdollahi, S.; Nadri, S. Mater. Sci. Eng. C 2016, 69, 447-452.

(14) Fan, D. W.; Bao, C. Z.; Khan, M. S.; Wang, C. L.; Zhang, Y.; Liu, Q. Z.; Zhang, X.; Wei, Q. Biosens. Bioelectron. 2018, 106, 14-20. 